\title{
Coercivity Values Enhancement by Incorporation of Magnetic Powders in Inorganic Matrix Hosts
}

\author{
J. R. Martínez ${ }^{1 *}$, J. Román de Alba ${ }^{1,2}$, I. G. Blanco-Esqueda ${ }^{2}$, A. Guerrero-Serrano ${ }^{2,3}$, \\ G. Ortega-Zarzosa ${ }^{3}$ \\ ${ }^{1}$ Departamento de Físico-Matemáticas, Universidad Autónoma de San Luis Potosí, San Luis Potosí, México; ${ }^{2}$ Centro de Investi- \\ gación en Materiales Avanzados S.C., Chihuahua, México; ${ }^{3}$ Facultad de Ciencias, Universidad Autónoma de San Luis Potosí, San \\ Luis Potosí, México. \\ Email: ${ }^{*}$ flash@fciencias.uaslp.mx
}

Received August 22 ${ }^{\text {nd }}, 2012$; revised September 22 ${ }^{\text {nd }}, 2012$; accepted September $30^{\text {th }}, 2012$

\begin{abstract}
Enhancement in coercivity values of precursor powders of cobalt ferrite embedded in silica xerogel as well as polyaniline was observed using vibrating sample magnetometry. We compared the magnetic properties of pure precursor powders of ferrite cobalt prepared by coprecipitation method and those embedded in xerogel and polyaniline matrix, prepared by sol-gel and by a conventional in situ chemical oxidation polymerization, respectively. The main magnetic effect is the altered coercivity value growing two magnitude orders for the precursor powders of cobalt ferrite embedded in silica xerogel and in polyaniline. The value goes from 52 Oe to 2200 Oe and 1054 Oe for pure coprecipitated precursor powder and embedded in silica xerogel, and embedded in polyaniline, respectively, without any heat-treatment.
\end{abstract}

Keywords: Magnetic Material; Nanocomposite; Xerogels; Polyaniline

\section{Introduction}

Inorganic matrixes, as host for nanoparticles, can be an effective medium to incorporate particles into with uniform size and determinated morphology having control over the homogeneous dispersion of ultrafine clusters of metal oxides. Different systems dispersed in insulating matrices, like silica, have been fabricated by sputter deposition, ball milling, evaporation, and by chemical methods [1-4].

Nanoparticles dispersed in insulating matrixes presented considerable changes in their properties when compared with their counterpart pure, bulk material.

In previous works we have reported that incorporation of materials in amorphous matrix prepared by sol-gel route promotes a reduction in the particle size, situation that governs the properties of the formed composite. In particular, M-Ba hexaferrites embedded in a $\mathrm{SiO}_{2}$ matrix present coercivity values which are significantly larger than those presented by pure coprecipitated M-Ba ferrite and of samples obtained directly by the sol-gel method [5]. This improvement in the coercivity can be ascribed to a reduction of the sizes of the particles.

Systems as barium titanate embedded also in silica xerogel matrix present similar results with a grain size

${ }^{*}$ Corresponding author. reduction. In this case a consequence of grain size reduction is the diminish of Curie temperature for the powder embedded in silica xerogel in comparation with the pure powders [6].

Sol-gel process has some advantages in making inorganic composite materials containing highly dispersed magnetic fine particles; the process facilitates a good and homogeneous dispersion of the particles into the inorganic matrix.

Magnetic nanoparticles present dramatic changes in the magnetic properties derived from their low dimensions; derived from these properties their principal applications are magnetic recording, medical diagnosis, drug deliver, sensor, etc. Magnetic nanocomposites have been produced in a great variety of matrixes such silicon oxide, aluminum oxide, porous glasses, and polymeric matrix.

Polyaniline (PANI) is known as one of the most technologically important materials because of its high conductivity, ease of preparation, good environmental stability, and a large variety of applications, especially in light-emitting and electronic devices, chemical sensors, separation membranes, and antistatic coating [7]. In recent years, there has been an increasing interest in the synthesis and design of conducting polymer-ferrite nanocomposites. Polymer magnetic nanocomposites represent a class of functional materials that have potential appli- 
cations in high density information storage media, wave absorbers, optical-magnetic media, cell separation, medical diagnosis, and controlled drug delivery [8].

These situations can be used to take advantage to prepare materials based in magnetic materials for eventually design efficient magnetic and electro-optical based solgel and polymer magnetic nanocomposites encapsulated biomolecules. For this, it is necessary to understand the structural evolution of magnetic compounds embedded in inorganic matrix such as silica xerogel matrix, and PANI, as well as the interaction between the particles embedded with the inorganic matrix, and then correlate this with its magnetic properties.

Previous results indicate us that among, the chemical process in the formation of the matrix, an interaction beetwen the particles embedded and the matrix formed ocurrs, producing alteration over the structure of the particles embedded and in the structure of the matrix, for which is posible produce devitrification or partial crystalization, and then induce changes in the physical and chemical properties of the composite.

In order to focus on the interaction process between the compound embedded ant the particular matrix, we deal in this paper with the magnetically study of precursor powders of Co-ferrite embedded in silica xerogel matrix and in PANI matrix. Special attention is pointed out in the coercitivity values caused by the adopted experimental conditions. We found that due to the interacttion process a enhancement of the corcitivity values are obtained in two magnitude orders for the precursor powders of cobalt ferrite embedded in silica xerogel and in polyaniline. We present only the magnetic comportment. Detailed analyses between structural and magnetic properties are in process.

\section{Experimental Method}

Precursor powders of Co-ferrite prepared by chemical co-precipitation method were embedded in a silica xerogel matrix prepared by the sol-gel method as well as in polyaniline matrix prepared by in situ polymerization method.

To prepare the ferrite cobalt precursor powder, a solution of $\mathrm{FeCl}_{3} \cdot 6 \mathrm{H}_{2} \mathrm{O}$ and $\mathrm{CoCl}_{2} \cdot 6 \mathrm{H}_{2} \mathrm{O}$, in water, was poured into $\mathrm{NaOH} / \mathrm{HNO}_{3}$, alkaline solution, under a molar ratio of $\mathrm{Co}(\mathrm{II}) / \mathrm{Fe}(\mathrm{III})$ of 0.5 and $\mathrm{pH}$ of 12 . The obtained powder was embedded in a silica xerogel matrix as well as in a polyaniline matrix.

The process to embed the precursor powder of cobalt ferrite was as follow, the starting solutions for the silica xerogels composites were prepared by mixing tetraethyl orthosilicate (TEOS), water, and ethanol. The molar ratios of ethanol to TEOS and water to TEOS were 4:1 and 11.67:1, respectively.
In order to obtain the final composites of $\mathrm{CoFe}-\mathrm{SiO}_{2}$, arbitrary amounts of Co-Fe were added to the ethanol. A homogenous solution of all components was obtained by mixing for about 15 minutes using a magnetic stirring. Soft pieces of the gels were obtained after about $48 \mathrm{hr}$. Those pieces were ground to form a fine powder.

PANI/(Co ferrite precursor nanocomposites) were prepared by a simple in situ polymerization method. In a typical procedure, a certain amount of cobalt ferrite precursor powder were added to $100 \mathrm{~mL}$ of $0.1 \mathrm{M} \mathrm{HCl}$ solution containing $0.91 \mathrm{~mL}$ of aniline monomer and stirred for $30 \mathrm{~min}$. The $1.58 \mathrm{~g}$ of $\mathrm{KMnO}_{4}$ in $8.24 \mathrm{~mL}$ of $0.1 \mathrm{M}$ $\mathrm{HCl}$ solution was then added dropwise to the suspension mixture with a constant stirring. The polymerization was allowed to proceed for $3 \mathrm{~h}$ at a temperature of $0^{\circ} \mathrm{C}$. The nanocomposites were obtained by filtering and washing the suspension with deionized water and $\mathrm{HCl}$, and dried in air at $60^{\circ} \mathrm{C}$ for $24 \mathrm{~h}$.

Both set of samples as well as the pure coprecipitated precursor powder of cobalt ferrite were analysed by vibrating sample magnetometry (VSM) to determine the magnetic properties obtained. The magnetic measurements were performed using Micromag 2000 vibrating sample magnetometer, at room temperature with maximum applied field of 10 KOe. We look at the variations of the coercivity field when the powders are embedded on $\mathrm{SiO}_{2}$ and PANI matrix. The ratio $\mathrm{Mr} / \mathrm{Ms}$ wich is a measurement of the magnetic hardeness also were obtained for all samples. The hysteresis loops were normalized in order to compare the magnetic behaviour, the magnetic saturation, wich depends of the quantity of magnetic materials in the composite, was not studied in this work.

To obtain the cobalt ferrite is necessary to anneal the sample above $600^{\circ} \mathrm{C}$. The precursor powders used in this study were obtained without annealing, in order to produce posibble chemical changes of the unstable magnetic phase of the precursor powders due to the interactions process and analyze the magnetic properties of the products which can be different thus such pure cobalt ferrite powders embedded on $\mathrm{SiO}_{2}$ and PANI matrix.

\section{Results and Discussion}

Figure 1 shows the hysteresis loops of the pure coprecipitated precursor powder of cobalt ferrite, sample with the powder embedded in the silica xerogel matrix, and, the sample with the powder embedded in the PANI matrix. The hysteresis loop of the powders without sintering indicates that cobalt ferrite is not formed yet. Regularly, to obtain the cobalt ferrite be coprecipitation technique is necessary to anneal the powders above $600^{\circ} \mathrm{C}$. The powders, when are embedded in a PANI matrix, shows an increased in coercivity about $1.05 \mathrm{KOe}$, but when these un-sintering powders are embedded in a silica xerogel 




Figure 1. Hysteresis loops of the pure precursor Co-ferrite, embedded in silica xerogel matrix, and embedded in PANI.

matrix, the coercivity reaches up a value of about 2.2 KOe.

According to the particle size for the pure precursor Co-ferrite $(\sim 9 \mathrm{~nm})$, we assumed that particles has a monodomain magnetic structure, then the increase in the coercivity of the embedded powders may be due to the interaction effect of the dispersed particles with the matrix. According to literature the increase in the coercivity is due to an increase in the dipolar interaction between particles [9]. In this case the interaction of the dispersed particles with the matrix encourages the dipolar magnetic interaction between particles.

The magnetic behavior of the precursor powders of cobalt ferrite shows the presence of more than one magnetic phases and non saturation in the magnetization. It is evident an interaction between the powder embedded and the matrix, silica xerogel as well as PANI matrix.

The presence of more than one phase is more evident when the sample is embedded in PANI matrix than when is embedded in silica xerogel. In the former case the saturation is obtained for an applied field of 2200 Oe.

The discontinuities in the hysteresis loops of the embedded powders can be explained as consequence of two magnetic phases due the cobalt ferrite is not formed yet, one phase may be correspond to an iron compound and the other magnetic phase is due a cobalt compound, evidentiated by the interaction process.

The obtained coercivity value for the Fe-Co: $\mathrm{SiO}_{2}$ composite was improved with respect to the value obtained for the pure coprecipitated precursor ferrite powder in two magnitude orders. This result is surprising because, in previous report an increase with a two factor were reported for $\mathrm{M}$-Ba ferrite embedded in silica matrix [5].

The coercivity values measurement are 52 Oe and
2200 Oe for pure coprecipitated precursor powder and embedded in silica xerogel, respectively.

Thus the interaction process between the powder embedded and the silica xerogel matrix conduce to obtain a magnetic composite with high coercivity.

The precursor ferrite-glass interface strongly influences the coercivity of the nanocomposite. In a previous work, we have reported that the grain size of barium hexaferrite particles, synthesized by the coprecipitation method, embedded in a silica xerogel matrix noticeably diminished with respect to the pure particle and then the obtained coercivity values were improved with respect to the values obtained from the pure coprecipitated hexaferrite sample $[5,10]$. Similar results have been reported for $\mathrm{M}-\mathrm{Sr}$ ferrite embedded in a $\mathrm{SiO}_{2}$ matrix [11]. The coercivity of this kind of pure ferrite materials is affected by defects, grain size, and grain distribution. The improvement in the coercivity has been ascribed to a reduction in the grain size of the embedded magnetic particles as well as to a reduction in the magnetic interaction of the magnetic particles. The improvement in coercivity has been reported with a factor of two.

In the present case, the improvement in coercivity is in two magnitude order. The surprising ever so much increase of the coercivity has been due to the low mean grain size of the precursor powders of cobalt ferrite which is about $9 \mathrm{~nm}$ according to the TEM image shown in Figure 2.

The grain size, maybe diminished when embedded in the silica xerogel matrix accord to the previous results, and the magnetic interaction between the embedded magnetic particles is reduced, increasing the coercivity value.

Figure 3 show X-ray diffractograms of the pure Co

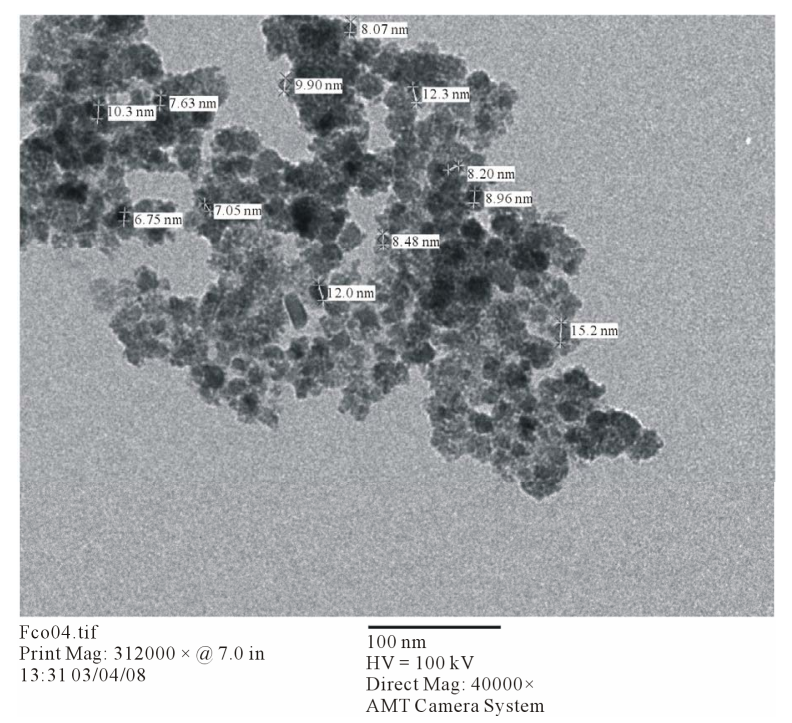

Figure 2. TEM image of the pure precursor powder of cobalt ferrite nanoparticles. 


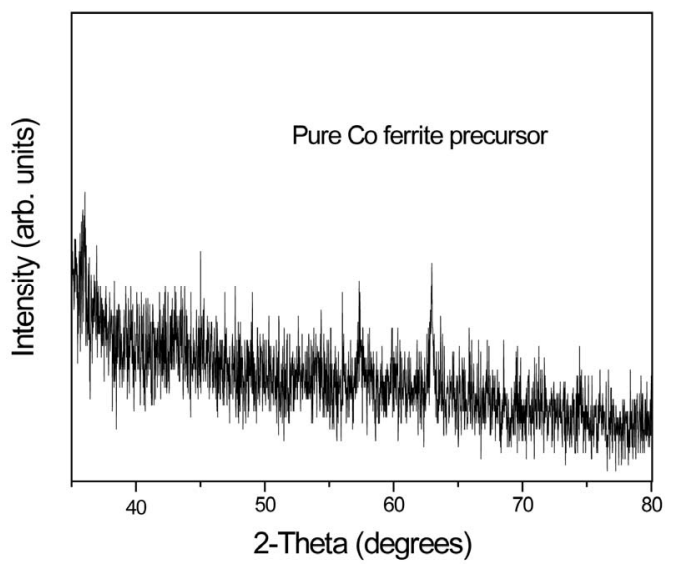

(a)

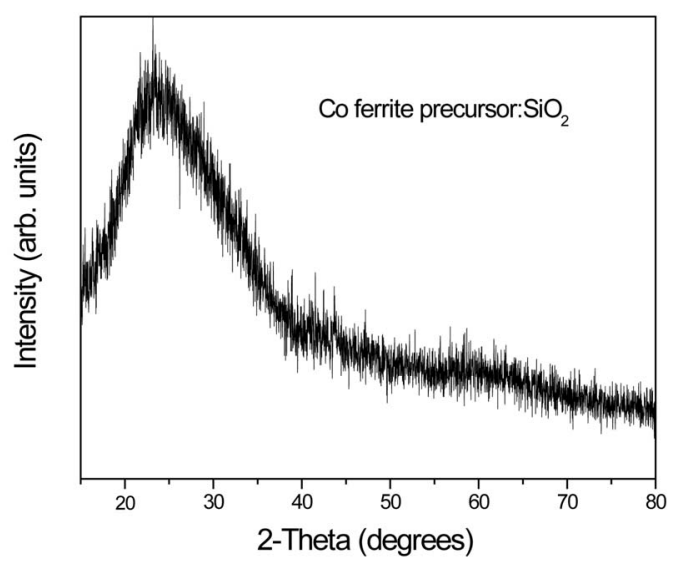

(b)

Figure 3. X-ray diffractograms of the (a) pure Co ferrite precursor powder and (b) sample with the powder embedded in the silica xerogel matrix.

ferrite precursor powder and sample with the powder embedded in the silica xerogel matrix in which we can observed that cobalt ferrite is not formed yet with presence of cobalt and iron oxides; the broad peak indicate very small grain size. The broad peak are pronunced in the sample with the powder embedded in the silica xerogel matrix.

For the precursor powder of cobalt ferrite embedded in PANI matrix the coercivity value grows notability from 52 Oe to 1054 Oe. This is a size effect, in which for fine nanoparticles conduce to a noticeable enhancement of the coercivity value. There have been several reports on the decrease of coercivity for the magnetic nanoparticles coated with nonmagnetic matrix when interparticle interaction have decreased, due to the increase of particleparticle separation, in particular polyaniline/M-Ba ferrite composites [12-15]. In our case, we have particles with very low size, and the concentration, although was arbitrary, was low, thus we have a dilution of the magnetic particles in a nonmagnetic matrix. Enhancement of the coercivity value has been observed for magnetic par- ticles, such $\gamma-\mathrm{Fe}_{2} \mathrm{O}_{3}$, partially reduced with polyphosphate surface $[16,17]$ and coated with a Co layer [18].

The dilution of the magnetic particles in a nonmagnetic matrix produces an enhancement of coercivity, by reducing the dipole-dipole interaction [14,19], verified by some works for which is observed a coercivity enhancement of $50 \%[20,21]$.

Thus the low grain sizes of the precursor powder of cobalt ferrite as well as the low concentration produce a noticeable increase of two magnitude orders for the coercivity value when the former powder was embedded in xerogel and PANI matrix. Furthermore the possible reduction of the grain size for the precursor powder of cobalt ferrite embedded in silica xerogel matrix, accord with previous results induce a coercivity enhancement of $4230 \%$

\section{Conclusions}

Using the vibrating sample magnetrometry analysis, the magnetic parameters of pure precursor powder of cobalt ferrite, and embedded in silica xerogel and PANI could be successfully determined. The main magnetic effect is the enhancement of the coercivity due to the interaction between the pure precursor powder of cobalt ferrite and the inorganic matrixes.

The coercivity value growing two magnitude orders for the precursor powders of cobalt ferrite embedded in silica xerogel and in polyaniline. The value goes from 52 Oe to 2200 Oe and 1054 Oe for pure coprecipitated precursor powder and embedded in silica xerogel, and embedded in polyaniline, respectively. This surprising growth is due to the small grain size of the precursor powder, which is about $9 \mathrm{~nm}$ and the dilution in the matrix systems, which reducing the dipole-dipole interacttion.

\section{Acknowledgements}

The authors thank Moises Gutiérrez Amaya and J.A. de la Cruz Mendoza for technical assistance.

\section{REFERENCES}

[1] C. L. Chien, "Magnetism and Giant Magneto-Transport Properties in Granular Solids," Annual Review of Materials Science, Vol. 25, 1995, pp. 129-160. doi:10.1146/annurev.ms.25.080195.001021

[2] G. Xiao, S. Liou, A. Levy, J. Taylos and C. L. Chien, "Magnetic Relaxation in Fe- $\left(\mathrm{SiO}_{2}\right)$ Granular Films," Physical Review B, Vol. 34, No. 11, 1986, pp. 7573-7577. doi:10.1103/PhysRevB.34.7573

[3] C. Estournes, T. Lutz, J. Happich, T. Quaranta, P. Wissler, J. Guill, "Nickel nanoparticles in Silica Gel: Preparation and Magnetic Properties,” Journal of Magnetism and Magnetic Materials, Vol. 173, No. 1-2, 1997, pp. 83-92. doi:10.1016/S0304-8853(97)00144-3. 
[4] L. Zhang, G. C. Papaefthymiou, R. F. Ziolo and J. Y. Ying, "Novel $\gamma$ - $\mathrm{Fe}_{2} \mathrm{O}_{3} / \mathrm{SiO}_{2}$ Magnetic Nanocomposites via Sol-Gel Matrix-Mediated Synthesis," Nanostructured Materials, Vol. 9, No. 1-8, 1997, pp. 185-188. doi:10.1016/S0965-9773(97)00049-4.

[5] S. Ponce-Castañeda, J. R. Martínez, F. Ruiz, S. A. Palomares-Sánchez and J. A. Matutes-Aquino, "Magnetic Properties Enhancement of M-Ba Ferrites Embedded in a $\mathrm{SiO}_{2}$ Matrix," Journal of Magnetism and Magnetic Materials, Vol. 250, 2002, pp. 160-163. doi:10.1016/S0304-8853(02)00374-8

[6] J. R. Martínez, J. A. de la Cruz-Mendoza, S. A. Palomares-Sánchez, G. Vázquez-García, G. Ortega-Zarzosa and F. Ruiz, "Grain Size Reduction Effect of Barium Titanate Embedded in Silica Xerogel,” Materials Letters, Vol. 62, No. 17-18, 2008, pp. 2947-2949. doi:10.1016/j.matlet.2008.01.081

[7] J. R. Ellis, "Competing Materials to Intrinsically Conducting Poltmers and Their Applications” In: T. A. Skotheim, Ed., Handbook of Conducting Polymers, Marcel Dekker, New York, 1986, pp. 501-505.

[8] J. Alam, U. Riaz and S. Ahmad, "Effect of Ferrofluid Concentration on Electrical and Magnetic Properties of the $\mathrm{Fe}_{3} \mathrm{O}_{4}$ /PANI Nanocomposites,” Journal of Magnetism and Magnetic Materials, Vol. 314, No. 2, 2007, pp. 93-99. doi:10.1016/j.jmmm.2007.02.195

[9] K. H. J. Buschow, "Magnetic Superconductor Materials," 2nd Edition, Elsevier, Amsterdam, 2005.

[10] S. A. Palomares-Sánchez, S. Ponce-Castañeda, J. R. Martínez, Y. M. Chumakov, F. Leccabue, B. E. Watts and R. Salazar-Ortiz, "Structural Analysis of Barium Hexaferrite Embedded in an Amorphous Matrix,” Materials Letters, Vol. 60, No. 8, 2006, pp. 1076-1079. doi:10.1016/j.matlet.2005.10.112

[11] S. Peng, J. F. Hu, H. W. Qin, et al., "Preparation and Magnetic Properties of M-Sr Ferrites Embedded in a $\mathrm{SiO}_{2}$ Matrix,” Rare Metal Materials and Engineering, Vol. 34, No. 7, 2005, 1151-1153.

[12] J. Jiang, L.-H. Ai, D.-B. Qin, H. Liu, and L.-C. Li, “Preparation and Characterization of Electromagnetic Functionalized Polyaniline/ $\mathrm{BaFe}_{12} \mathrm{O}_{19}$ Composites," Synthetic Metals, Vol. 159, No. 7-8, 2009, pp. 695-699. doi:10.1016/i.synthmet.2008.12.021
[13] J. Jiang, L. Li, F. Xu, "In Situ Synthesis and Characterization of $\mathrm{LiNi}_{0.5} \mathrm{La}_{0.08} \mathrm{Fe}_{1.92} \mathrm{O}_{4}$-Polyaniline Core-Shell Nanocomposites," Journal of Physics and Chemistry of Solids, Vol. 68, No. 9, 2007, pp. 1656-1662. doi:10.1016/j.jpcs.2007.04.007

[14] X. Battle and A. Labarta, "Finite-Size Effects in Fine Particles: Magnetic and Transport Properties," Journal of Physics D: Applied Physics, Vol. 35, No. 6, 2002, pp. R15. doi:10.1088/0022-3727/35/6/201

[15] W. Luo, S. R. Nagel, T. F. Rosenbaum and R. E. Rosenweig, "Dipole Interactions with Random Anisotropy in a Frozen Ferrofluid,” Physical Review Letters, Vol. 67, No. 19, 1991, pp. 2721-2724. doi:10.1103/PhysRevLett.67.2721

[16] F. Itoh and M. Satou, "Finite-Size Effects in Fine Particles: Magnetic and Transport Properties," Japanese Journal of Applied Physics, Vol. 14, 1975, pp. 2091-2092. doi:10.1143/JJAP.14.2091

[17] F. E. Spada, F. T. Parker, C. Y. Nakakura and A. E. Berkowitz, "Studies of Anisotropy Mechanisms in Polyphosphate-Treated Magnetic Iron Oxide Particles," Journal of Magnetism and Magnetic Materials, Vol. 120, No. 1-3, 1993, pp. 129-135. doi:10.1016/0304-8853(93)91304-P

[18] F. E. Spada, F. T. Parker, A. E. Berkowitz and T. J. Cox, " $H_{K}$ Distributions and $H_{C}$ Calculations for Magnetic Recording Particles,” Journal of Applied Physics, Vol. 75, No. 3, 1994, pp. 5562-5570. doi:10.1063/1.355689

[19] H. N. Bertram and A. Bhatia, "The Effect of Interactions on the Saturation Remanence of Particulate Assemblies," IEEE Transactions on Magnetics, Vol. 9, No. 2, 1973, pp. 127-133. doi:10.1109/TMAG.1973.1067574

[20] M. P. Morales, M. J. Muñoz-Aguado, J. L. García- Palacios, F. J. Lázaro and C. J. Serna, "Coercivity Enhancement in $\gamma-\mathrm{Fe}_{2} \mathrm{O}_{3}$ Particles Dispersed at Low-Volume Fraction," Journal of Magnetism and Magnetic Materials, Vol. 183, No. 1-2, 1998, pp. 232-240. doi:10.1016/S0304-8853(97)01061-5

[21] F. T. Parker, A. E. Barkowitz and S. B. Slade, " $H_{c}$ Enhancement of Co-Adsorbed $\gamma-\mathrm{Fe}_{2} \mathrm{O}_{3}$ Particles via Surface Treatment with Sodium Polyphosphate," Journal of Applied Physics, Vol. 75, No. 10, 1994, pp. 1681-1683. doi:10.1063/1.356354 